\title{
Chapter 2 \\ Documentation and Recent Initiatives \\ on the Use of Simulation in the Context of POD
}

- Matzkanin, G. \& Yolken, T., A Technology Assessment of Probability of Detection (POD) for Nondestructive Evaluation (NDE), NTIAC, 2001

In this report Matzkanin and Yolken present a review of the early efforts towards the use of simulation for determining POD curves for different NDE methods until 2001.

The efforts since that date have mostly been supported through two initiatives: the MAPOD working group in the USA and the European project PICASSO. We will briefly describe these two initiatives.

\section{- MAPOD working group}

MAPOD working group, active from 2004 to 2011, gathered several entities in the United-States in order to promote the increased understanding, development and implementation of model-assisted POD methodologies.

More information about MAPOD working group and documents that it produced can be found at http://www.cnde.iastate.edu/mapod/index.htm.

\section{- PICASSO project}

PICASSO was a research collaborative project registered in the Seventh Framework Program of the European Commission from 2009 to 2012. It gathered several industrial partners of the aeronautics industry and research centers. The aim of the PICASSO project was to build a new and original concept of "POD curves based on NDT simulation in addition to existing experimental data base".

More information about PICASSO project can be found at http://www.picassondt.eu/. 